\title{
On Design and Simulation of Electrostatic Sensor Used for Measuring Gas-Solid Two-phase Flow
}

\author{
Zhigen Yu \\ School of Mechanical-electronic and Automobile Engineering, Huzhou Vocational and Technical \\ College, Huzhou 313000, China
}

252849772@qq.com

Keywords: gas-solid two-phase flow, electrostatic sensor, finite element analysis.

Abstract. By analyzing the current theory model of electrostatic sensor, we present an improved one that based on point charge. The sensing mechanism is described precisely because influencing factors such as the geometry of sensing element, the tubing insulating material, the geometry of electro-magnetic shielding around are considered synthetically in this model. A reasonable structure if finally got after simulated by analysis software of the finite element, and electrostatic sensor designed according to this model is widely used to measure the density of gas-solid two-phase flow.

\section{Introduction}

The gas-solid two-phase flow is a common logistics mold in production process. Pulverized coal, cement, ore, salts, flour are all conveyed by pipeline in industries of power, building material, metallurgy, environmental sanitation, medicines and grain processing. It becomes more and more important to measure precisely the parameters of gas-solid two-phase flow with the high demands on detecting parameters and control. For instance, it has become an important study to control the pollution sources by detecting and controlling dust emitted from industries. The conventional method to measure the speed and density of gas-solid two-phase flow is to construct a computing model by deriving from experimental analyze and introducing a correction factor ${ }^{[1]}$. It has also become a forward subject concerned by scholars at home and abroad because the research on multi-phase flow is constrained by the technology of measuring the gas-solid two-phase or multi-phase flow ${ }^{[2]}$.

\section{An Improved Model of Measuring Electrostatic Sensor Based on Point Charge}

Three methods are used to detect the electrostatic particle: triboelectricity, electrodynamics, electrostatic. There are fine differences in expressing the sensing principles. In triboelectricity, particles are charged by friction or their contact or collision with sensing probes. While in electrodynamics, dynamic characteristics of measuring mechanism are emphasized, and only those moving charged particles affect the sensor's output. The electrostatics emphasizes the characteristics of electrostatic induction and thus is well suitable for the name. Fig. 1 shows the system model for measuring parameters of gas-solid two-phase flow that based on particle staticelectricity. Its fundamental principle is that particle staticelectricity is detected by a charge-converted circuit according to the different charging mechanism of the tested objects. Electrical signals are output to measuring instrument after being amplified and filtered. Hence, parameters of gas-solid two-phase flow are detected online.

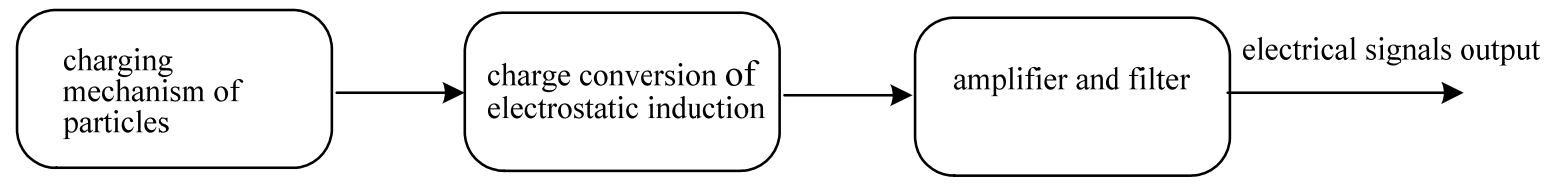

Figure 1. diagram of electrostatic sensor for measuring gas-solid two-phase flow

The current sensing elements has two structures, one is contact (ring-shaped), the other is non-contact (rod-like). The sensor electrodes are required to have good conductive ability ${ }^{[3]}$. The 
ring-shaped electrostatic sensor is an ideal one used to detect parameters of multi-phase flow in industrial production and will be introduced in the following.

\subsection{Research Status of the Ring-shaped Model of Measuring Electrostatic Sensor}

The essence of the ring-shaped electrostatic sensor is to employ its interaction with the electrostatic field formed by charged particles. The outputting induced charges and potential vary with the electrostatic field because of the moving particles. It is necessary to build its physical and mathematical model in order to verify the interaction between charged particles and sensors, to understand its sensing mechanism deeply, to study quantitatively the sensing characteristics such as spatial sensitivity, spatial filtering effect, and frequency response, to provide theoretical basis necessary for determining and evaluating its performance, optimizing design, and improving its dynamic performance. Many models are built by scholars such as Gajewski from Technical University of Wroclaw in Poland ${ }^{[4]}$, YanYong ,Cheng from Tesside university in U.K [5]., and Murnane, Woodhead from University of Greenwich ${ }^{[6]}$. Although these models have been widely applied in researching on measuring parameters of gas-solid two-phase flow, there are massive assumed conditions in building models of dynamic mechanism, and both the spatial filtering characteristic and the frequency bandwidth characteristics are given.

Harsh conditions such as equipotental particles are required in Gajeski's model. In fact, it's impossible for non-conducting particles to be equipotental. Furthermore, the mutual-capacitance and self-capacitance are different for particles in different sensing area. So even for particles with same electricity but in different space, the induced potential in electrodes will change, which is not considered in this model. It's also impossible that in pneumatic conveying system, the particles are steady flow and disperse uniformly in pipeline. Form multiphase fluid mechanics, we know that even dispersed particles have different concentration in local space. Besides, charges carried by particles are different in every possible way. So the relation between charge's density and induced potential couldn't show the reaction between electrostatic field and electrode. More importantly, Gajeski didn't take into account the sensor's dimension that affects its performance in fact. To sum up, the model could only explain quantitatively the relation between the induced potential and the charges carried by particles or volume charges density. Its output affect by spatial distribution couldn't be analyzed without the particle's sensitivity distribution.

\subsection{An Improved Model of Measuring Electrostatic Sensor}

Models showed above describe the sensing mechanism in some extent, and provide theoretical basis for analyzing characteristics. There are too many assumed conditions in building the models and only axial length are taken into account. In practice, the electrostatic sensor is composed of sensing elements, insulated tube and electro-magnetic shielding. All of them should be taken into account in analyzing sensor's characteristic or in building mathematical models. Besides, when charged particles flow through electrode, equal but opposite charges are induced in the internal and external surface of the electrode. Charges induced are sum of those produced by particles' charges in the induction zone. So a single particle charge could be thought of as a point charge. The electrostatic sensor model here is built on the basis of point charge, and employs Gajeski's structure. As shown in Figure. 2, it could stand high pressure and is electric insulated because the insulated tube is made up of quartz glass. For modeling conveniently, let's assume that point charges in radial position move axially at a constant speed; ignore the magnetic effect of moving charges; assume that the particle charges that flow through the electrode is saturated, that the transmission pipeline a metal one with good conductivity and is grounded, that the electrode is a mental ring with extremely good conductivity and shunt capacitance is ignored. This model is more suitable for pneumatic transportting pulverized coal, cement and etc. 


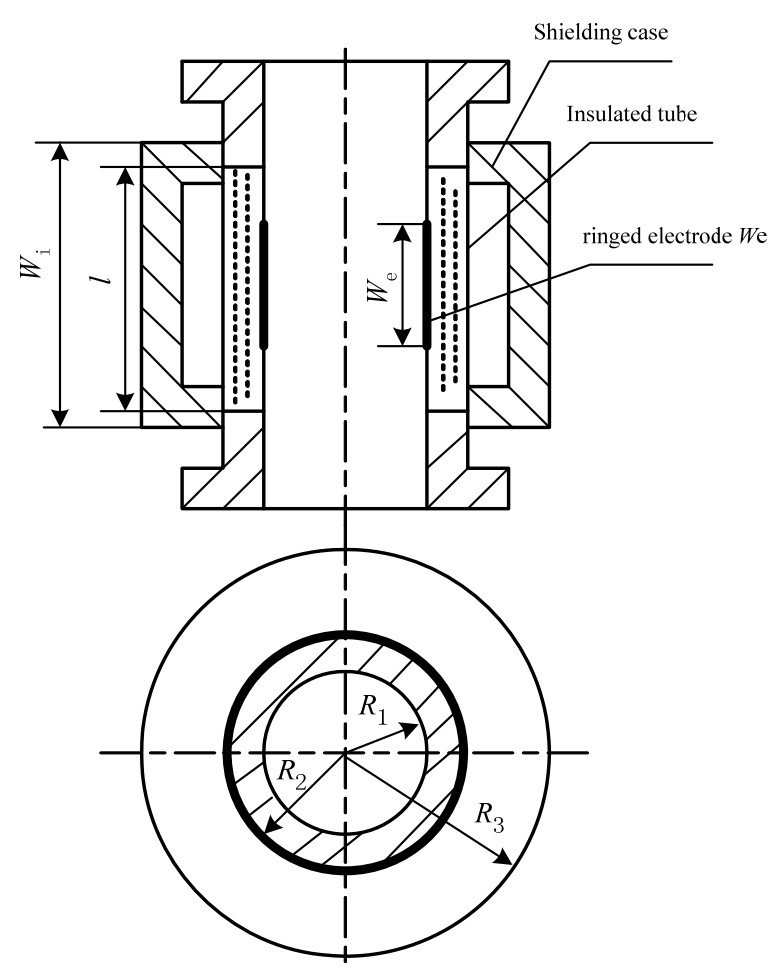

Figure 2. structure model of an improved electrostatic sensor based on point charge

Because the interaction between the field formed by inducing charges and that formed by induced charges, conductor could keep electrostatic equilibrium in very short time $(10-19 \mathrm{~s})^{[7]}$. The interaction between the moving point charges and the electrostatic sensor can be described by electrostatic field. When point charges are in the sensing zone, the electrostatic field satisfies the following Poisson equation and the boundary condition:

$$
\left\{\begin{array}{l}
\nabla(\varepsilon(x, y, z) \nabla \phi(x, y, z))=-\rho(x, y, z) \Phi(x, y, z) \\
\left.\Phi(x, y, z)\right|_{(\mathrm{x}, \mathrm{y}, \mathrm{z}) \in \Gamma_{p}}=0 \\
\left.\Phi(x, y, z)\right|_{(\mathrm{x}, \mathrm{y}, \mathrm{z}) \in \Gamma_{S}}=0 \\
\left.\Phi(x, y, z)\right|_{(\mathrm{x}, \mathrm{y}, \mathrm{z}) \in \Gamma_{e}}=\text { Cons }
\end{array}\right.
$$

According to Gauss theorem, density of the induced charges in the electrode's surface is:

$$
\sigma(x, y, z)=\vec{D}(x, y, z)=\varepsilon(x, y, z) \vec{E}(x, y, z)=-\varepsilon(x, y, z) \bullet \nabla \phi(x, y, z)
$$

Where, $\phi(x, y, z)$ is a field potential with the unit of $\mathrm{V} ; \rho(x, y, z)$ is a density distribution of the volume charges with the unit of $\mathrm{C} / \mathrm{m} 3 ; \varepsilon(x, y, z)$ is a dielectric constant distribution with the unit of $\mathrm{F} / \mathrm{m} ; \Gamma_{p} 、 \Gamma_{S}$ are boundaries formed by pipeline and shielding; $\Gamma_{e}$ is an electrode boundary; Cons means that the sensor electrode is equipotential; $\sigma(x, y, z)$ is a density distribution of induced charges in electrode with the unit of $\mathrm{C} / \mathrm{m} 3 ; D(x, y, z)$ is an electric displacement vector near the electrode inner wall with the unit of $\mathrm{C} / \mathrm{m} 2, E(x, y, z)$ is a electric field distribution with the unit of $\mathrm{V} / \mathrm{m}$.

The quantity Q of induced charges in the electrode's inner surface with area of S is computed by the Equation (2):

$$
Q=\int_{S} \sigma(x, y, z) \bullet d s
$$

From the mathematical model above, we can seen that in a sensitive space, under conditions of the known $\varepsilon(x, y, z), \rho(x, y, z)$, and boundary, the quantity $\mathrm{Q}$ of induced charges can be computed by Equation (1), (2) and (3). Because of the complex boundary condition and flow in pipeline, it is relatively difficult to parse the mathematical model and the induced charges are computed by finite element simulation. 


\section{Finite Element Simulation of Sensing Element}

With graphic interface, program structure, and interactive graphic processing, ANSYS software can be used for computing structure, fluid, thermal, electromagnetic, and coupled field, which alleviates workloads of modeling, finite element solution, results' analysis and evaluation. For electric field, we can analyze current's conduction, circuits, field-circuit coupling, and electrostatic field. Electrical quantities such as current density, electric field intensity, and capacitance can be solved as well [7]. ANSYS8.1 software of PC version, together with ANSYS APDL, is used to program sensitivity distribution in sensing field of the electrostatic sensor. And parameters such as structure ones, potential inside the sensing field, induced electric, sensitivity can be analyzed, simulated, and evaluated.

\subsection{Finite Element Analyzing Electrostatic Field of the Sensing Elements in Electrostatic Sensor}

When charged particles move in the pipeline, induced charges will be produced in the electrode and the metal pipeline, and there will be an interaction between the electrostatic field formed by induced charges and that formed by charged particles. So, the induced charges distribution in electrode and the induced electricity will be affected by the electrode and pipeline inevitably. Because the interaction between the field formed by inducing charges and that formed by induced charges, conductor could keep electrostatic equilibrium in very short time (10-19s). The field can thought of as an electrostatic one according to the criterion of near steady in physics. At present, there are five methods used to solve the modeling: analytical method, boundary finite element method, finite difference method, boundary finite element method, and Monte Carlo method. Which one is chosen just depends on geometry's complexity and dimension of problems to be solved, and software suitable. Approximate solutions will be got in finite difference method. With the development of electronics and computer technology, the finite difference method has been widely applied in solving problems in electromagnetic field such as electrostatic field, time-varying field, and nonlinear field etc. The best advantage of it is that it fits for problems with complex boundary and complicated media distribution, and is not limited by the boundary shape. Hence, the finite difference method is selected to analyze its sensing characteristics and to design its sensing elements optimally.

Problems in electrostatic field can be turned into not only definite problems of differential equation, but also variational problems in which extreme values are to be solved. These two are equivalent according to Variational Principle. Based on Variational Principle, the finite element method developed form difference scheme. First, boundary value problems expressed in differential equation is turned into variational problems that getting extreme values. Secondly, the continuous field is discretized by field subdividing. A finite element subspace is constructed to turn approximately the variational problems into solving extreme value of multivariate function [8]. According to Thomason theorem, charge distribution in the surface of charge conductor enables the electrostatic field to have minimum energy. And equation (4) is gotten:

$$
\left\{\begin{array}{l}
J_{\varphi}=\iiint_{D} \frac{\varepsilon(x, y, z)}{2}\left\{\left[\frac{\partial \varphi(x, y, z)}{\partial x}\right]^{2}+\left[\frac{\partial \varphi(x, y, z)}{\partial y}\right]^{2}+\left[\frac{\partial \varphi(x, y, z)}{\partial z}\right]^{2}-\rho \varphi\right\} d x d y d z=\min \\
\left.\Phi(x, y, z)\right|_{(\mathrm{x}, \mathrm{y}, \mathrm{z}) \in \Gamma_{p}}=0 \\
\left.\Phi(x, y, z)\right|_{(\mathrm{x}, \mathrm{y}, \mathrm{z}) \Gamma_{S}}=0 \\
\left.\Phi(x, y, z)\right|_{(\mathrm{x}, \mathrm{y}, \mathrm{z}) \in \Gamma_{e}}=\text { Cons }
\end{array}\right.
$$

Where, $\mathrm{D}$ is the shielding inner space.

\subsection{Finite Element Model of Electrostatic Sensor}

Let the axial direction of the electrode be Z-axis, the radial be r-axis, and the circumferential be $\theta$-axis in the global coordinate system. Two electrostatic field distributions that formed by point charges at any site of the infinite grounding cylinder: one is a two-dimension field when point charges at the axis, the other is a three-dimension filed when charges deviate from the axis. The finite element 
analysis method is quick enough for two-dimension field but slow for three-dimension filed. The three-dimension filed can be converted to two-dimension in conditions of that per point charge is supposed to distribute uniformly at the charge's radial circumference. Both the pipeline itself and constraint formed by liner charge are axisymmetric. Although electrostatic field formed by liner charges is different form that formed by point charges, they are equivalent for induced charges in the electrode because electrostatic fields at the same axial and radial position have the same induced electricity by Superposition Principle. Modeling thus becomes dramatically easy and computing is speeded up.

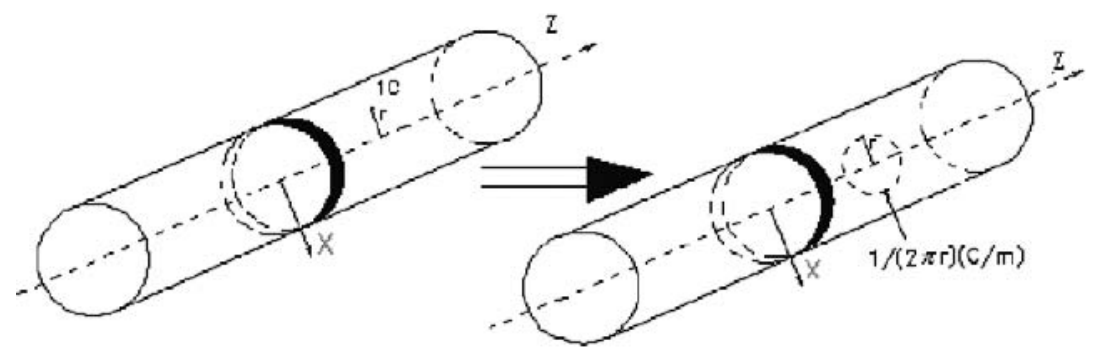

Figure 3 Simplifying three-dimension electrostatic filed to two-dimension one

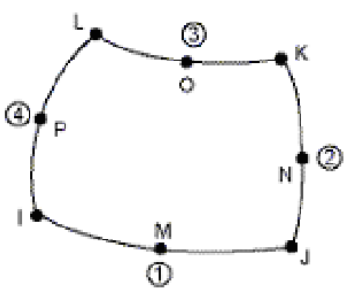

Figure 4 PLANE121 geometry

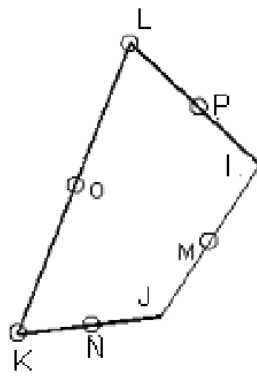

Figure 5 INFINE110's geometry

From the analysis above we know that point charge at any position can be solved according to a two-dimension field. The PLANE121 (shown in Fig.4) which has a two-D quadrilateral with eight nodes or triangle axisymmetrical unit is selected according to the probe's size and shape of and the demanding computing precision. In a far-field area, the INFIN110 (shown in Fig.5) which is an axisymmetrical unit with a two-D quadrilateral eight nodes matches the PLANE121. Besides, the electrostatic sensor is divided into three areas: the inner insulated pipe, the wall of insulated pipe, and the shielding space between the outer pipe and the shielding case. A dense grid is divided because there is a drastic changing potential near the point charges, electrode, and the shielding case. Fig. 6 shows the axisymmetrical grid division of point charges locate in the center of the axis and Fig. 7 shows that of point charges off the axis center.

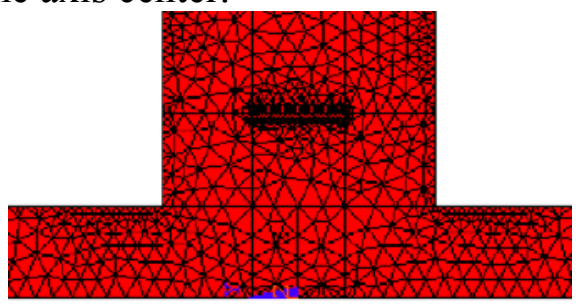

Figure 6 Grid division of the finite element model with point charges in the center of the axis

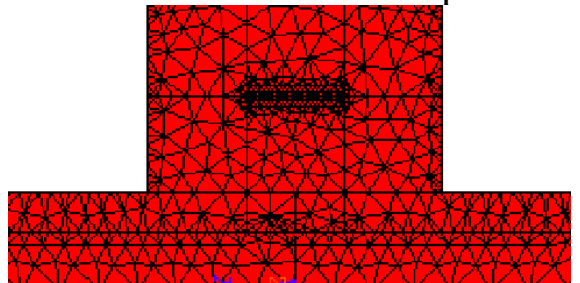

Figure 7 Grid division of the finite element model with point charges off the center of the axis

\subsection{Analyzing Results of Simulation Experiment}

We draw several useful conclusions based on simulation experiment on the sensing elements: 
1) A longer axial length (We) results in higher sensitivity, a more homogeneous distribution of the section's sensitive field, and a wider sensitive axial space. A too long electrode doesn't enables the sensor to response to the high frequency signal of the fluid space because of the special filtering effect of the electrostatic sensor, while a too low electrode results in a small output, a low signal-to-noise ratio, and a difficult detection.

2) The tube-wall's thickness (R2-R1) and its relative dielectric constant (cri). When the thickness satisfies mechanical strength, the thinner, the better. A thick tube-wall lows the absolute sensitivity because it occupies the high sensitive area. However, an increased thickness contributes to the uniformity of the section's sensitivity. An increased relative dielectric constanteri increases its absolute sensitivity and a non-uniform sensitivity of the section, but almost hasn't any influence on the sensitivity of the axial space.

3) Radial size of the shielding case (R3-R2) and axial length (L). The radial size of the shielding case has less influence on the sensitivity and its distribution. But the absolute sensitivity of the central section increased with the axial length, which leads to an increasing the average sensitivity and contributes to the uniformity of the section's sensitivity. An increased axial length results in a high sensitivity and a wide sensitive space. That is, an appropriately increased axial length makes circuit design easier.

\section{Conclusion}

On basis of analyzing the merits and demerits of the electrostatic sensor models, we give an improved model which thinks of the probe made up of sensor electrodes, insulated pipe and electromagnetic shielding as a whole. By ANSYS8.1 (an infinite element analysis software) of PC version and APDL, an infinite element program is composed to computer the sensitivity distribution of the electrostatic sensor. Feasible scheme is provided as well to modeling and to research on the characteristic of the sensor for measuring two-phase flow.

\section{Acknowledgements}

Zhi-gen Yu (1965-), an associate professor in Huzhou Vocational and Technological College, a visiting scholar of Zhejiang University, majoring in researching in electrical power and electron. Tel: $0572-2363418$.

\section{References}

[1] Y L Zhou, R Yan and D Wang. Type electrostatic sensor array based on gas-solid two-phase flow velocity detection. Science technology and engineering, 36(2016)42-47.

[2] Z Gan, F Q Shao and Q H Li. Based on electrostatic sensor cross-correlation gas/solid two phase flow velocity measurement. Petroleum chemical industry of institutions of higher learning, 6(2012)78-82.

[3] Y Yan and J Ma. Measurement of Particulate Velocity under Stack-flow Condition. Meas.Sci. Technol., 2000, 11:59-65.

[4] G Zhe, X L Wang and Q H Li, etc. Oil field power system small current single-phase earth fault line selection research. Journal of liaoning university of petroleum chemical industry, 2(2013):67-69.

[5] J LI, C L XU, S M Wang. Type electrostatic sensor array based on autocorrelation of the particle velocity measurement. Journal of engineering thermal physics, 7(2014)1343-1347.

[6] Zushou Zhang, On Magnitude Estimation of Time for Conductors to Reach Electrostatic Equilibrium.Physics and Engineering, (12)2013, 20-21.

Reference to a book:

[7] Shuguang Gong, Ansys Operation Command and Parametric Programming. Beijing: Mechanical Industry Press, 2004: 231. 